\title{
ORIENTACIÓN AL MERCADO Y TIPOLOGÍA DE LAS \\ COOPERATIVAS AGROALIMENTARIAS EN BASE A LA \\ COMPETITIVIDAD. CASO-ESTUDIO DE LAS CITRÍCOLAS ESPAÑNAS
}

\author{
POR \\ Natalia LAJARA CAMILLERI y \\ Ricardo J. SERVER IZQUIERDO ${ }^{1}$
}

\section{RESUMEN}

La globalización ha recrudecido más si cabe la competencia en los mercados y las empresas deben afianzar y mejorar su posición competitiva para asegurar la supervivencia. Para ello resulta vital, entre otras cuestiones, velar por los intereses de los inversores al tiempo que cuidan de sus clientes. Las cooperativas sufren una coyuntura semejante respecto a sus socios y el mercado, es decir respecto a sus clientes internos (socios) y externos (mercado). El enfoque de las actividades y la estrategia de la cooperativa frente a esta dualidad determina su grado de orientación al mercado.

Los estudios relacionados con la orientación al mercado en cooperativas son escasos en comparación con otras formas empresariales y han estado normalmente centrados en la figura de las cooperativas de segundo grado y circunscritos a sectores muy específicos.

El sector citrícola español es uno de los más dinámicos y desarrollados del panorama productivo agrario. Su extensa experiencia comercial tanto a nivel doméstico como internacional confiere un carácter diferenciador a las entidades que operan en este subsector. Este trabajo analiza la posición de las cooperativas citrícolas españolas en relación a la orientación al mercado así como los factores de gestión y estrategia que están relacionados

\footnotetext{
${ }^{1}$ CEGEA. Universitat Politècnica de València. Direcciones de correo electrónico: nalade@cegea.upv.es y rjserver@esp.upv.es.
}

REVESCO N 121 - Segundo Cuatrimestre 2016 - ISSN: 1885-8031 - www.ucm.es/info/revesco

http://dx.doi.org/10.5209/rev_REVE.2016.v121.51305

Fecha de recepción: 22/07/2015

Fecha de aceptación: 07/09/2015 
con ella. Para ello se utiliza una escala MARKOR, validada en estudios anteriores, sobre una muestra de 45 cooperativas.

Se establece además una tipología de cooperativas citrícolas en base a los factores de competitividad que permiten ahondar en el conocimiento que existe sobre esta cuestión en entidades de economía social. Los resultados ponen de manifiesto un elevado grado de relación entre la innovación, el perfil del empresario y el conocimiento del entorno en relación con el grado de orientación al mercado.

Palabras clave: cooperativas agroalimentarias, factores de competitividad, escala MARKOR, análisis cluster, ANOVA.

Claves ECONLIT: M100; O300; L22; P13.

\title{
MARKET ORIENTATION AND TYPOLOGY OF AGRIFOOD COOPERATIVES ACCORDING TO COMPETITIVENESS. CASE-STUDY OF SPANISH CITRUS COOPERATIVES
}

\begin{abstract}
Globalization has intensified competition more so in markets, therefore companies have to strengthen and improve their competitive position to ensure survival. To this end, it is crucial, among other issues, to protect the interests of investors while caring for their customers. In the case of cooperatives, this situation is reflected in their internal customers (members) and their external customers (markets). The approach to activities and the strategy to deal with this duality determines the degree of market orientation.
\end{abstract}

Studies related to market orientation in cooperatives are relatively scarce and have frequently been focused in federative cooperatives and very specific sectors.

The Spanish citrus sector is one of the most dynamic and developed in the agricultural productive context. Its vast marketing experience both in domestic and foreign markets makes the difference in the companies that deal in this subsector. This paper analyzes the position of Spanish citrus cooperatives in relation to market orientation as well as to management and strategy factors that can be related to it. A previously validated MARKOR scale is used on a 45 cooperative sample. 
It also establishes a typology of citrus cooperatives based on competitive factors. It permits to deepen the knowledge that exists on this issue on social Economy organizations.

The results show a high degree of relationship between innovation, manager profile and the market knowledge in relation to the degree of market orientation.

Keywords: agrifood cooperatives, factors of competitiveness, MARKOR scale, cluster analysis, ANOVA.

\section{INTRODUCCIÓN}

La globalización y la progresiva liberalización del comercio con terceros países han incrementado de forma notable la competencia en los mercados. En el sector agroalimentario este hecho viene marcado por ciertos condicionantes tales como los cada vez más exigentes controles de calidad, la influencia climática en los resultados de las producciones y el carácter perecedero de los productos (Juliá et al., 2010).

El sector productivo agroalimentario en España cuenta con una importante presencia de cooperativas que actúan como vehículo concentrador de oferta en la comercialización, tratando de capturar valores añadidos, además de ofrecer ventajas a sus socios en la adquisición de insumos (Juliá y Marí, 2002).

Actualmente en España existen 3.397 cooperativas agroalimentarias que proporcionan 97.944 empleos directos y cuentan con una facturación conjunta de 25.696 millones de euros (OSCAE, 2013). El peso de la producción comercializada a través de esta fórmula empresarial ha ido incrementándose de forma sostenida en los últimos años hasta alcanzar en la actualidad el 60\% del valor de la Producción Final Agraria española (MAGRAMA, 2012) y el 30\% del valor de la Producción Bruta de la Industria Alimentaria Española (FIAB, 2013).

Dentro del sector agroalimentario cabe destacar la relevancia del cultivo y exportación de cítricos en España. Este subsector ha sido tradicionalmente uno de los puntales de la agricultura española así como la balanza comercial. España ocupa la posición de máximo exportador de cítricos en fresco, siendo el origen del $23 \%$ de las naranjas que se exportan a nivel mundial y del 37\% de las mandarinas (FAOSTAT, 2013).

Las cooperativas, por su tradición y su implantación en el Levante español, son agentes protagonistas en esta situación pero no escapan de la realidad del mercado y deben 
afrontar los retos con los que se encuentran, tanto los que comparten con el resto de operadores como los que son específicos de esta forma jurídica.

A fin de asegurar su supervivencia las cooperativas deben ser capaces de reforzar su competitividad, desarrollando y mejorando aspectos de estructura y de gestión que ayuden a fortalecer su posición en el mercado. Con este fin se han determinado los factores de competitividad que contribuyen al éxito de las cooperativas agroalimentarias. Tal como afirman Marí et al (2013), los principales pilares de la competitividad en la empresa cooperativa son: innovación, internacionalización, dimensión, formación, diversificación y orientación al mercado.

La mayoría de las cuestiones anteriores han sido abordadas en la literatura científica pero habitualmente basando los estudios en empresas de tipo capitalista. Si bien es cierto que existen publicaciones que analizan específicamente estos aspectos en su aplicación en cooperativas, se trata en su mayor parte de estudios empíricos que no han permitido alcanzar conclusiones extrapolables al sector.

El objetivo de este trabajo es contribuir al conocimiento en este sentido, planteando el estudio de la orientación al mercado en cooperativas agroalimentarias españolas, analizando específicamente aquellas que se dedican a la comercialización de cítricos. El objetivo es doble, por una parte se determina el grado de relación que existe entre la orientación al mercado y algunos elementos de la estructura y la gestión de las empresas. Una vez establecidos estos vínculos, el segundo objetivo se centra en llevar a cabo una clasificación de las cooperativas de la muestra que permita establecer grupos con características de gestión comunes. Para ello, tras una revisión bibliográfica de trabajos previos y el establecimiento de un marco teórico que sirva de base para la investigación, se describe la metodología empleada y los resultados obtenidos.

\section{MARCO TEÓRICO E HIPÓTESIS DE INVESTIGACIÓN}

\subsection{La orientación al mercado en cooperativas}

La orientación al mercado ha sido abordada por ciertos autores como una vertiente del marketing (Deshpandé et al., 1993) si bien existe un enfoque divergente, situándola más bien como un elemento de cultura empresarial, una parte fundamental de la estrategia de la empresa (Vázquez et al., 1999). 
Las raíces de la orientación al mercado se sitúan en los años 60 con la aportación de Felton, no obstante es en la década de los noventa cuando esta idea cobra auge. Por una parte Narver y Slater (1990) propusieron el concepto como una filosofía o cultura organizacional que se apoya en tres comportamientos: la orientación al consumidor, la orientación al competidor y la coordinación interfuncional. Simultáneamente Kohli y Jaworski (1990) propusieron bajo la misma denominación un concepto basado en la generación de inteligencia de mercado, la diseminación de dicha información entre los departamentos y la respuesta conjunta por parte de toda la organización.

Tal como sostiene Rivera (1999), “la orientación al mercado es una estrategia que la organización usa para mantener la ventaja competitiva sostenible”. Para ello la orientación al mercado se basa en la selección de los mercados que la empresa quiere satisfacer y en el adecuado uso de la información de forma interna en la organización.

En definitiva, una organización orientada al mercado es aquella que da prioridad a la generación de valor percibido por parte de su público objetivo para asegurar su supervivencia. Para ello asume como filosofía de gestión integral el concepto de marketing, traduce orgánicamente esta filosofía en forma de cultura organizativa de negocio y aplica operativamente el concepto (Álvarez et al, 2001).

La medición del grado de orientación al mercado ha sido objeto de controversia a nivel académico con las aportaciones fundamentales de Narver y Slater con la escala MKTOR y de Kohli, Jaworski y Kumar con su escala MARKOR. Ambas escalas han sido ampliamente utilizadas en multitud de estudios empíricos, realizando pequeñas adaptaciones de acuerdo al sector analizado.

El interés que suscita este concepto en cooperativas se debe a la existencia de dos clientes, los clientes internos (socios) y los externos (mercado). El enfoque de las actividades y la estrategia de la cooperativa frente a esta dualidad determina su grado de orientación al socio, a la venta o al mercado (Senise, 2003).

Los estudios relacionados con la orientación al mercado en cooperativas son relativamente escasos y han estado normalmente centrados en la figura de las cooperativas de segundo grado y circunscritos a sectores muy específicos. En España Senise (2003) y Montegut et al (2007) analizan la orientación al mercado en cooperativas oleícolas en Andalucía y Cataluña, respectivamente. Cambra ha tratado la cuestión de la orientación al 
mercado en cooperativas vitivinícolas (Cambra et al, 2011; Cambra y Fuster, 2004; Cambra y Fuster, 2005) y Arcas (2002) aborda la relación entre las cooperativas de primer grado asociadas en una entidad de segundo grado y cómo la integración mejora la orientación al mercado. Hernández-Espallardo y Arcas (2003) estudian sobre una muestra de cooperativas cómo crear y estimular este factor de competitividad en base a las relaciones que se establecen entre las cooperativas de segundo grado y sus asociadas.

A nivel internacional, Kyriakopoulos y Van Bekkum (1999) y posteriormente Kyriakopoulos, Meulenberg y Nilsson (2004) estudian en cooperativas agroalimentarias la orientación al mercado en relación a distintos aspectos de la gestión como la estructura organizativa, la estrategia de mercado o las políticas de precios. Amadieu y Viviani (2010) indirectamente incluyen la cuestión de la orientación al mercado en el estudio de empresas vitivinícolas francesas, buscando una relación entre inversión en intangibles y rendimiento en términos comerciales. Finalmente, destacar el análisis mediante la técnica del caso llevado a cabo por Grunert et al (2005), en él se valora el grado de orientación al mercado en cuatro cadenas de valor agroalimentarias incluyendo y analizando el papel de la cooperativa Danish Crown dentro de la comercialización de bacon. Existen por tanto todavía muchas incógnitas pendientes de resolver sobre la orientación al mercado en cooperativas.

\subsection{Hipótesis de investigación}

La innovación ha sido objeto de estudio en los últimos años en relación con la orientación al mercado (Narver y Slater, 1990; Grinstein, 2008, Gómez et al, 2009, entre otros) al incluir a ésta última como uno de sus factores internos. De hecho, es considerada como un antecedente del proceso de innovación, ya que las organizaciones orientadas al mercado requieren innovación para ser capaces de mantener sus ventajas competitivas (Cambra et al, 2011). Es más, al monitorizar las necesidades de los clientes se induce el proceso innovador. Han et al. (1998) registraron que la orientación al mercado influía positiva y significativamente tanto en las innovaciones técnicas como en las administrativas. MaydeuOlivares y Lado (2003) señalaron que la innovación es un elemento catalizador en la relación entre la orientación al mercado y el rendimiento en las empresas.

No obstante, los trabajos realizados han estado normalmente referidos a empresas mercantiles y son escasos los estudios empíricos basados en cooperativas. Cabe recordar que la cooperativa, por su estructura de propiedad, sufre de una importante dualidad en su 
orientación al considerar al socio también como cliente (Juliá et al, 2009). No obstante, en referencia a cooperativas, Bijman (2010) señala que la orientación al mercado fuerza a operar de forma más estratégica, empujando hacia la innovación. En este sentido, dentro del ámbito agroalimentario español, Cambra et al (2011) llevan a cabo un ambicioso estudio sobre una muestra de 71 bodegas vitivinícolas -algunas de ellas cooperativas- inscritas en las denominación de origen de Aragón (España). Sus resultados confirman la relación positiva entre la orientación al mercado y la innovación.

Este trabajo trata por tanto de proporcionar evidencia empírica sobre esta relación, específicamente en la figura de la cooperativa agroalimentaria.

\section{H1: Las entidades orientadas al mercado muestran mayor propensión a ser innovadoras.}

La orientación al mercado se identifica en muchas ocasiones como una "cultura de organización" (Del Río y Varela, 2006). Esta conceptualización como filosofía de gestión establece un nexo con el perfil directivo de la organización, por ello es susceptible de análisis la relación que exista entre las características de rigidez, aversión al riesgo, agresividad empresarial, etc. con el grado de orientación al mercado que presenta una empresa.

En efecto, Gupta y Govindarajan (1984) apuntan que en concreto la disposición a asumir riesgos (willingness to take risks) de los directivos de las empresas favorece las estrategias de expansión mediante nuevos productos o a través de nuevos mercados. Esta afirmación ha sido contrastada en numerosas ocasiones tanto en el plano de la internacionalización de la empresa como en la innovación desarrollada.

El bajo nivel de profesionalización que se observaba en la dirección de las cooperativas agroalimentarias españolas (García y Aranda, 2001) es posiblemente uno de los elementos que ha retrasado el estudio de la influencia de las características personales del directivo en las estrategias empresariales de las cooperativas. A pesar de existir excelentes análisis sobre el nivel de formación en las cooperativas (Mozas y Rodríguez, 2003; Marí et al., 2013, entre otros), no hay evidencias sobre el efecto que la aversión al riesgo o la agresividad empresarial del gerente pueden tener sobre la actividad en cooperativas.

La agresividad empresarial se entiende como una cierta capacidad de anticipación por parte del empresario (Del Río y Varela, 2006). Mide por tanto la proactividad del directivo ante el contexto socioeconómico en el que la empresa (cooperativa) opera. En base a lo 
anterior parece oportuno abordar esta cuestión y su influencia en concreto en el grado de orientación al mercado de la cooperativa.

\section{H2: Los directivos de las empresas con mayor orientación al mercado son más agresivos, en términos empresariales, que los de las empresas menos orientadas al mercado.}

Las dimensiones de la orientación al mercado (generación de la información, diseminación de la inteligencia y respuesta conjunta) propuestas por Kohli y Jaworski (1990) establecen un punto de partida para asumir que las empresas con una mayor orientación al mercado se encuentran en una posición aventajada en la búsqueda de información sobre el mercado. En este sentido Martín Armario et al. (2008) plantean esta premisa y demuestran la relación al estudiar una muestra de PYMEs andaluzas con actividad internacional, basándose en los hallazgos de Tuominen et al (1997) y Jaworski y Kohli (1996) donde se establece que las empresas orientadas al mercado son empresas orientadas al aprendizaje.

De forma similar se pretende plantear un estudio concerniente a empresas cooperativas, analizando si existe relación entre ambas cuestiones.

\section{H3: Las cooperativas con mayor orientación al mercado tienen un mayor grado de conocimiento de mercados, clientes y competencia.}

La relación entre el tamaño empresarial y otros factores de competitividad es una cuestión recurrente en los estudios de management. La orientación al mercado, como elemento integrador de la cultura empresarial, no precisa de un tamaño de empresa mínimo para poder formar parte de la empresa, no obstante Liu (1995) sintetiza la controversia existente en la literatura académica alrededor de esta cuestión y aporta evidencias sobre una muestra de empresas del Reino Unido y concluye que las empresas de mayor tamaño muestran una mayor orientación al mercado.

Por el contrario González-Benito et al. (2015) señalan, tras analizar una muestra de 440 empresas españolas de diferentes tamaños, que el tamaño empresarial no causa la relación entre orientación al mercado, emprendimiento e innovación pero sí que actúa como variable moderadora en algunos aspectos.

En base a ello parece oportuno contrastar esta relación en el ámbito cooperativo, específicamente en el sector citrícola. 
H4: Las cooperativas con mayor orientación al mercado son normalmente de mayor tamaño empresarial (en términos de empleados, balance, facturación).

\section{METODOLOGÍA}

\subsection{Métodos e identificación de variables}

El análisis de la varianza (ANOVA) es una técnica de dependencia que mide las diferencias en una variable métrica basada en un conjunto de valores categóricos (no métricos) que actúan como predictores (Hair et al., 1999). El ANOVA en definitiva analiza la probabilidad de que las diferencias detectadas se deban únicamente al error muestral.

Como técnica de análisis ha sido ampliamente utilizada en estudios de corte económico-empresarial. Longinos et al. (2012) utilizan el ANOVA para determinar la existencia o no de diferencias significativas en distintos aspectos como la transparencia o el gobierno corporativo tomando como referencia el grado de cumplimiento de responsabilidad social en una muestra de cooperativas agrarias y de trabajo asociado. Meliá-Martí y MartínezGarcía (2015) aplican pruebas no paramétricas para determinar la existencia de diferencias significativas en los resultados obtenidos por una muestra de cooperativas antes y después de llevar a cabo un proceso de fusión.

Guzmán et al. (2014) estudian la existencia de diferencias significativas entre los clústers de empresas (entre ellas, cooperativas) obtenidas tras realizar un análisis de conglomerados. La técnica multivariante aplicada en el trabajo, el análisis de conglomerados o clúster, forma parte del grupo de métodos de interdependencia. Se trata de herramientas que permiten identificar la estructura entre una serie de variables dado un conjunto de individuos. A partir de esta identificación es posible realizar descripciones o incluso descubrir nuevas relaciones (Hair et al, 1999).

El análisis clúster agrupa de forma exploratoria a los individuos basándose en las características que poseen. Los grupos obtenidos muestran un elevado grado de homogeneidad interna al tiempo que se diferencian significativamente del resto de grupos. En este sentido, pretende dar cumplimiento al objetivo del trabajo de establecer una tipología dentro de las cooperativas de la muestra en base a los factores de competitividad señalados.

La variable orientación al mercado -OM- ha sido definida utilizando la adaptación de la escala MARKOR para cooperativas realizada por Arcas (2002). Esta escala consta de tres 
bloques principales: generación de inteligencia, respuesta de toda la empresa y diseminación de la inteligencia. La escala consta de 20 ítems que se puntúan de 1 a 5 (ver Anexo) por lo que el rango de la variable es de 0-100. La variable ha sido sometida a un proceso de categorización (Tabla 1) a fin de agrupar las respuestas obtenidas debido al reducido número de individuos de la muestra. La conversión se ha realizado tratando que los intervalos formados expliquen la mayor proporción de varianza de la variable numérica. En este caso, un 93,66\%. Los grupos se definen utilizando técnicas de análisis clúster, de forma que la varianza intragrupos sea la menor posible y se mantenga la varianza intergrupos.

Tabla 1. Categorización de la variable "orientación al mercado"

\begin{tabular}{|l|r|r|}
\hline & N & Categoría \\
\hline Menos de 61 & 7 & 1 \\
Entre 61 y 76 & 8 & 2 \\
Entre 76 y 85 & 9 & 3 \\
Más de 85 & 15 & 4 \\
\hline
\end{tabular}

Fuente: Elaboración propia

Las variables independientes se han definido atendiendo a las hipótesis de investigación planteadas (Tabla 2).

Tabla 2. Variables utilizadas

\begin{tabular}{|c|c|c|c|}
\hline Bloque & $\begin{array}{l}\text { Nombre } \\
\text { variable }\end{array}$ & Descripción & Fuente \\
\hline \multirow[t]{3}{*}{ Dimensión } & FACT & $\begin{array}{l}\text { Facturación en el último ejercicio. Escala: } 1 \\
(\text { menos de } 1 \mathrm{M} €), 2 \text { (1-2M€), } 3(2-10 \mathrm{M} €), 4 \text { (10- } \\
43 \mathrm{M} €), 5(43-50 \mathrm{M} €), 6 \text { (más de } 50 \mathrm{M} €) \text {. }\end{array}$ & \multirow[t]{3}{*}{$\begin{array}{l}\text { Adaptación de Cavusgil } \\
\text { (1984), Cavusgil (1982) } \\
\text { Bilkey y Tesar (1977), }\end{array}$} \\
\hline & ACTIVO & $\begin{array}{l}\text { Valor del activo en el último ejercicio. Escala: } 1 \\
\text { (menos de } 1 \mathrm{M} €), 2 \text { (1-2M€), } 3 \text { (2-10M€), } 4 \text { (10- } \\
43 \mathrm{M} €), 5(43-50 \mathrm{M} €), 6 \text { (más de } 50 \mathrm{M} €) \text {. }\end{array}$ & \\
\hline & EMPLEADO & $\begin{array}{l}\text { Número de empleados. Escala: 1(menos de 10), } 2 \\
(10-50), 3(50-250), 4 \text { (más de 250). }\end{array}$ & \\
\hline Perfil exportador & PESO_EXP & $\begin{array}{l}\text { Propensión o intensidad exportadora. Ventas } \\
\text { extranjero/Ventas totales. Escala: } 1 \text { (menos } 10 \%) \text {, } \\
2(10-25 \%), 3(25-50 \%), 4(50-75 \%), 5 \text { (más del } 75 \%)\end{array}$ & 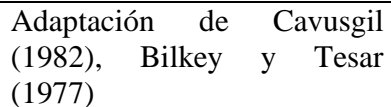 \\
\hline \multirow{3}{*}{$\begin{array}{l}\text { Conocimiento } \\
\text { mercados } \\
\text { exteriores }\end{array}$} & CON_CLI & $\begin{array}{l}\text { Grado de conocimiento sobre } \\
\text { exteriores. Escala 5-Likert }\end{array}$ & \multirow[t]{3}{*}{ Martín Armario (2003) } \\
\hline & CON_ENT & $\begin{array}{l}\text { Grado de conocimiento sobre entorno de su } \\
\text { principal mercado exterior. Escala 5-Likert }\end{array}$ & \\
\hline & CON_COMP & $\begin{array}{l}\text { Grado de conocimiento sobre la competencia en } \\
\text { su principal mercado exterior. Escala 5-Likert }\end{array}$ & \\
\hline
\end{tabular}




\begin{tabular}{|c|c|c|c|}
\hline $\begin{array}{l}\text { Orientación al } \\
\text { mercado }\end{array}$ & $\mathrm{OM}$ & $\begin{array}{l}\text { Se realizan encuentros entre los distintos } \\
\text { departamentos para discutir tendencias del } \\
\text { mercado }\end{array}$ & $\begin{array}{l}\text { Arcas (2002), He y Wei } \\
(2011) \text {, Martín Armario } \\
(2003) \text {, Martín Armario et al } \\
(2008)\end{array}$ \\
\hline \multirow{3}{*}{$\begin{array}{l}\text { Perfil } \\
\text { empresario }\end{array}$} & RIGIDEZ & Rigidez empresarial, resistencia al cambio & \multirow[t]{3}{*}{ Del Río y Varela (2006) } \\
\hline & AUSE_AG & Ausencia de agresividad empresarial & \\
\hline & ACT_INT & $\begin{array}{l}\text { Actitud ante la internacionalización de la } \\
\text { empresa }\end{array}$ & \\
\hline \multirow[t]{9}{*}{ Innovación } & INNOV1 & $\begin{array}{l}\text { En la cooperativa se dispone de un sistema para } \\
\text { gestionar las ideas de mejora de los socios y } \\
\text { empleados Sí (1) No (2) }\end{array}$ & \multirow{9}{*}{$\begin{array}{l}\text { Dai et al (2014), Suh y Kim } \\
\text { (2014), Boso et al (2013), } \\
\text { Castaño-Martínez (2012), } \\
\text { Aragón y Rubio (2005) }\end{array}$} \\
\hline & INNOV2 & $\begin{array}{l}\text { En la cooperativa se dispone de un sistema para } \\
\text { gestionar las ideas de mejora de los clientes Sí } \\
\text { (1) No (2) }\end{array}$ & \\
\hline & INNOV3 & $\begin{array}{l}\text { El nivel de innovaciones de producto en los } \\
\text { últimos } 3 \text { años en la cooperativa lo considero... } \\
\text { Escala 5-Likert }\end{array}$ & \\
\hline & INNOV4 & $\begin{array}{l}\text { Los esfuerzos realizados en las innovaciones de } \\
\text { producto en términos de recursos considero que } \\
\text { son... Escala 5-Likert }\end{array}$ & \\
\hline & INNOV5 & $\begin{array}{l}\text { El nivel de innovaciones de proceso en los } \\
\text { últimos } 3 \text { años en la cooperativa lo considero... } \\
\text { Escala 5-Likert }\end{array}$ & \\
\hline & INNOV6 & $\begin{array}{l}\text { Los esfuerzos realizados en las innovaciones de } \\
\text { proceso en términos de recursos considero que } \\
\text { son... Escala 5-Likert }\end{array}$ & \\
\hline & INNOV7 & $\begin{array}{l}\text { El nivel de innovaciones de organización en los } \\
\text { últimos } 3 \text { años en la cooperativa lo considero... } \\
\text { Escala 5-Likert }\end{array}$ & \\
\hline & INNOV8 & $\begin{array}{l}\text { Los esfuerzos realizados en las innovaciones de } \\
\text { organización en términos de recursos considero } \\
\text { que son... Escala 5-Likert }\end{array}$ & \\
\hline & INNOV & $\begin{array}{l}\text { En conjunto considero a la cooperativa } \\
\text { innovadora Sí (1) No (2) }\end{array}$ & \\
\hline
\end{tabular}

Fuente: Elaboración propia

La dimensión empresarial es una variable que se construye a partir de la definición de micro, pequeña, mediana y gran empresa (por exclusión) recogida en la Recomendación 2003/361/CE, de 6 de mayo, sobre la definición de microempresas, pequeñas y medianas empresas. La categoría de pertenencia se determina en función del número de empleados (EMPLEADO), volumen de negocios anual (FACT) y balance total de cada empresa (ACTIVO).

La innovación en el ámbito empresarial presenta el reto de su medición, existiendo numerosas propuestas y, al tratarse de un concepto en permanente evolución, también la forma de cuantificarlo varía. En este estudio, siguiendo la medición realizada por Aragón y Rubio (2005), la variable INNOV recoge la clasificación como cooperativa innovadora o no (1/0) que realizan los gerentes o directores comerciales de las cooperativas de la muestra. Adicionalmente se han incluido una serie de indicadores secundarios (INNOV1-INNOV8) 
que, siguiendo con la escala propuesta por Aragón y Rubio (2005), tratan de detallar la naturaleza de la/s innovación/es en las organizaciones.

El conocimiento de los mercados exteriores es un concepto medido a través de tres indicadores, según aporta Martín Armario (2003). Puede diferenciarse el conocimiento específico de clientes, de las condiciones del entorno y de la competencia. En todos los casos este conocimiento se mide mediante escala Likert de 1 a 5.

En referencia al bloque de actitud del empresario/gerente se han incluido ítems que miden la rigidez empresarial (la resistencia a introducir cambios en la gestión de la organización), la agresividad empresarial, entendida como la capacidad de anticipación por parte del empresario (Del Río y Varela, 2006). Mide por tanto la proactividad del directivo ante el contexto socioeconómico en el que la empresa (cooperativa) opera. Adicionalmente se cuantifica la actitud hacia la internacionalización en la empresa.

\subsection{Selección de la muestra y caracterización}

El ámbito de estudio es España, por ello, a partir del listado de Organizaciones de Productores de Cítricos (OPCs) proporcionado por el Ministerio de Agricultura, Alimentación y Medio Ambiente de España en marzo de 2014, se han identificado las entidades cuya forma jurídica es la cooperativa, tienen su domicilio en España y se encuentran en activo, su número asciende a 63 cooperativas y constituyen la población del estudio.

La recogida de datos se ha realizado en base a un cuestionario auto-administrado, formado por preguntas de respuesta cerrada o semi-abierta, agrupadas en diversos bloques (perfil del encuestado, orientación al mercado, innovación e internacionalización).

El cuestionario ha sido contestado por el gerente o el director comercial de las cooperativas entre los meses de junio y septiembre de 2014.

Debido al reducido número de entidades no se ha utilizado ningún método de muestreo, el cuestionario ha sido enviado por vía postal a todas las cooperativas. Tras realizar cinco contactos (vía telefónica, postal y visita personal), se ha obtenido una tasa de respuesta del $71 \%$. La muestra está formada por 45 cooperativas, se considera una muestra representativa asumiendo un nivel de confianza del $95 \%$ con un porcentaje de error del $8 \%$. 
Las 45 cooperativas españolas que componen la muestra se localizan en un 86,7\% en la Comunidad Valenciana, correspondiendo a Murcia, Andalucía y Cataluña el resto de las entidades. Esta distribución plasma la realidad de la población en la que el $86 \%$ de las OPCs reconocidas son valencianas. Se trata en todos los casos de cooperativas de primer grado que trabajan fundamentalmente con cítricos, si bien un $24,4 \%$ declara comercializar también otros productos (fundamentalmente frutas no cítricas, con el objetivo de completar el calendario de comercialización y mejorar el rendimiento de las instalaciones).

La heterogeneidad de la muestra se pone de manifiesto en las variables de dimensión (FACT, ACTIVO y EMPLEADO), con entidades que facturan menos de un millón de euros y otras que declaran ventas anuales entre los 43 y 50 millones de euros (Tabla 3). No obstante, atendiendo exclusivamente al criterio del número de empleados, el $82,2 \%$ de las entidades se clasificarían como empresas medianas y un $17,8 \%$ serían grandes.

El marcado carácter exportador del cooperativismo citrícola queda patente a través del peso de las exportaciones en la facturación que se sitúa por término medio en más de un 50\%.

Tabla 3. Estadísticos descriptivos

\begin{tabular}{|l|r|r|r|r|r|}
\hline & N & Mínimo & Máximo & Media & DS \\
\hline FACT $^{1}$ & 42 & 1,00 & 6,00 & 3,1860 & 1,05234 \\
ACTIVO $^{1}$ & 35 & 1,00 & 4,00 & 2,9714 &, 82197 \\
EMPLEADO $^{2}$ & 45 & 1,00 & 4,00 & 2,9778 &, 69048 \\
PESO_EXP $^{3}$ & 38 & 1,00 & 5,00 & 4,5000 & 1,08429 \\
\hline
\end{tabular}

'Escala: 1 (menos de 1M€), 2 (1-2M€), 3 (2-10M€), 4 (10-43M€), 5 (43-50M€), 6 (más de 50M€)

${ }^{2}$ Escala: 1 (menos de 10), 2 (10-50), 3 (50-250), 4 (más de 250).

${ }^{3}$ Escala: 1 (menos 10\%), 2(10-25\%), 3 (25-50\%), 4(50-75\%), 5 (más del 75\%)

Fuente: Elaboración propia

\section{RESULTADOS}

\subsection{Análisis ANOVA}

Para dar cumplimiento al primer objetivo propuesto y contrastar las hipótesis planteadas en relación a los factores relacionados con la orientación al mercado se ha utilizado el análisis de la varianza, ANOVA. La hipótesis nula establecida es que no existen diferencias significativas en las variables consideradas según el tipo de cooperativa del que se 
trate, definida en base a la categorización del grado de orientación al mercado. En caso de incumplimiento de esta hipótesis, es decir, una vez detectado que existen diferencias estadísticamente significativas entre las medias de una variable de al menos dos de los niveles del factor, será preciso determinar mediante técnicas post-hoc entre qué grupos se producen tales diferencias y el carácter de las mismas.

En este caso el factor será el tipo de cooperativa en función del grado de orientación al mercado con cuatro niveles posibles $(1,2,3,4)$ y las variables serán las especificadas en el apartado 3.1.

El análisis de la varianza es una técnica en la que la falta de normalidad tiene en general poca influencia en los resultados obtenidos, se asume que éstos son sustancialmente válidos aunque los datos sean no normales. Es posible afirmar que el análisis de la varianza es una técnica robusta frente a desviaciones de la normalidad (Peña, 2010).

Sin embargo sí que reviste importancia el cumplimiento, o no, de la homocedasticidad ya que condiciona el método a utilizar en el análisis de la varianza. Para contrastar la hipótesis de igualdad de varianzas de las variables dependientes en cada uno de los niveles del factor se aplica el estadístico de Levene (Tabla 4). Los resultados obtenidos permiten afirmar que esta hipótesis se cumple para todas las variables consideradas a excepción de las variables INNOV, ACT_INT, INNOV1, INNOV2, INNOV7 y CON_CLI. 
Tabla 4. Prueba de homogeneidad de varianzas

\begin{tabular}{|l|r|r|r|r|}
\hline & \multicolumn{1}{|c|}{$\begin{array}{r}\text { Estadístico de } \\
\text { Levene }\end{array}$} & gl1 & gl2 & \multicolumn{1}{l|}{ Sig. } \\
\hline Dimensión & 1,376 & 3 & 41 &, $264^{*}$ \\
INNOV & 11,090 & 3 & 41 &, 000 \\
RIGIDEZ &, 538 & 3 & 41 &, $659^{*}$ \\
AUSE_AG & 1,269 & 3 & 41 &, $298^{*}$ \\
ACT_INT & 5,375 & 3 & 41 &, 003 \\
INNOV1 & 35,264 & 3 & 41 &, 000 \\
INNOV2 & 13,722 & 3 & 41 &, 000 \\
INNOV3 & 2,344 & 3 & 41 &, $087^{*}$ \\
INNOV4 &, 347 & 3 & 41 &, $791^{*}$ \\
INNOV5 &, 805 & 3 & 41 &, $498^{*}$ \\
INNOV6 &, 153 & 3 & 41 &, $927^{*}$ \\
INNOV7 & 3,184 & 3 & 41 &, 034 \\
INNOV8 & 1,446 & 3 & 41 &, $243^{*}$ \\
CON_CLI & 2,423 & 3 & 37 &, 081 \\
CON_ENT &, 848 & 3 & 37 &, $477^{*}$ \\
CON_COMP &, 342 & 3 & 37 &, $795^{*}$ \\
\hline
\end{tabular}

Nota: $* \mathrm{p}>0,05$ No se puede rechazar la $\mathrm{H}_{0}$ - Homocedasticidad

Fuente: Elaboración propia

Los resultados de la aplicación del modelo de análisis de la varianza con las variables especificadas que han demostrado cumplir la condición de homocedasticidad se muestran en la Tabla 5. Se comprueba que existen diferencias significativas entre las medias de al menos dos de los niveles definidos en la tipología de cooperativas en función de su orientación al mercado en las variables relativas al perfil del gerente (RIGIDEZ y AUSE_AG), en la innovación (INNOV3, INNOV4, INNOV5 e INNOV8) y el conocimiento del mercado exterior (CON_COMP). Los resultados obtenidos con la prueba no paramétrica de KruskalWallis confirman la información obtenida a través del ANOVA. 
Tabla 5. Análisis de la varianza y pruebas no paramétricas

\begin{tabular}{|c|c|c|c|c|c|c|c|c|}
\hline & & \multicolumn{5}{|c|}{ ANOVA } & \multicolumn{2}{|c|}{ Kruskal-Wallis } \\
\hline & & $\begin{array}{l}\text { Suma de } \\
\text { cuadrados }\end{array}$ & $\mathrm{gl}$ & $\begin{array}{c}\text { Media } \\
\text { cuadrática }\end{array}$ & $\mathrm{F}$ & Sig. & Chi cuadrado & Sig \\
\hline \multirow{4}{*}{ Dimensión } & Inter-grupos &, 859 & 3 &, 286 &, 512 & ,676 & 1,511 & 0,680 \\
\hline & Intra-grupos & 22,919 & 41 & ,559 & & & & \\
\hline & Total & 23,778 & 44 & & & & & \\
\hline & Inter-grupos & 6,939 & 3 & 2,313 & 2,661 &, $061^{*}$ & 9,731 & $0,021 * *$ \\
\hline \multirow[t]{3}{*}{ RIGIDEZ } & Intra-grupos & 35,639 & 41 &, 869 & & & & \\
\hline & Total & 42,578 & 44 & & & & & \\
\hline & Inter-grupos & 11,522 & 3 & 3,841 & 3,170 &, $034 * *$ & 8,944 & $0,030 * *$ \\
\hline \multirow[t]{3}{*}{ AUSE_AG } & Intra-grupos & 49,678 & 41 & 1,212 & & & & \\
\hline & Total & 61,200 & 44 & & & & & \\
\hline & Inter-grupos & 14,138 & 3 & 4,713 & 5,044 &, $005 * *$ & 10,346 & $0,016 * *$ \\
\hline \multirow[t]{3}{*}{ INNOV3 } & Intra-grupos & 38,306 & 41 & ,934 & & & & \\
\hline & Total & 52,444 & 44 & & & & & \\
\hline & Inter-grupos & 9,730 & 3 & 3,243 & 3,010 &, $041 * *$ & 7,237 & $0,065^{*}$ \\
\hline \multirow[t]{3}{*}{ INNOV4 } & Intra-grupos & 44,181 & 41 & 1,078 & & & & \\
\hline & Total & 53,911 & 44 & & & & & \\
\hline & Inter-grupos & 11,165 & 3 & 3,722 & 2,754 &, $055^{*}$ & 7,205 & $0,066^{*}$ \\
\hline \multirow[t]{3}{*}{ INNOV5 } & Intra-grupos & 55,413 & 41 & 1,352 & & & & \\
\hline & Total & 66,578 & 44 & & & & & \\
\hline & Inter-grupos & 8,653 & 3 & 2,884 & 1,973 & ,133 & 5,345 & 0,148 \\
\hline \multirow[t]{3}{*}{ INNOV6 } & Intra-grupos & 59,925 & 41 & 1,462 & & & & \\
\hline & Total & 68,578 & 44 & & & & & \\
\hline & Inter-grupos & 6,388 & 3 & 2,129 & 2,524 &, $071^{*}$ & 7,035 & $0,071^{*}$ \\
\hline \multirow[t]{3}{*}{ INNOV8 } & Intra-grupos & 34,590 & 41 &, 844 & & & & \\
\hline & Total & 40,978 & 44 & & & & & \\
\hline & Inter-grupos & 2,852 & 3 &, 951 & 1,552 & ,217 & 6,568 & $0,087^{*}$ \\
\hline \multirow[t]{3}{*}{ CON_ENT } & Intra-grupos & 22,660 & 37 & ,612 & & & & \\
\hline & Total & 25,512 & 40 & & & & & \\
\hline & Inter-grupos & 4,877 & 3 & 1,626 & 2,399 &, $083^{*}$ & 7,173 & $0,067^{*}$ \\
\hline \multirow[t]{2}{*}{ CON_COMP } & Intra-grupos & 25,075 & 37 & ,678 & & & & \\
\hline & Total & 29,951 & 40 & & & & & \\
\hline
\end{tabular}

Fuente: Elaboración propia

Para las variables heterocedásticas se aplica el test de Brown-Forsythe (Tabla 6), sin embargo debido a la limitación de datos de la muestra en la mayoría de casos no es posible obtener resultados ya que en al menos uno de los grupos formados la varianza es cero, por lo que no se puede detectar la existencia o no de diferencias significativas en el caso de las variables INNOV, INNOV1 e INNOV2. Sin embargo, a través de la prueba no paramétrica de 
Kruskal-Wallis sí que es posible explorar relación entre estas variables con la orientación al mercado. Tanto en el caso del carácter innovador o no de la cooperativa (INNOV) como la existencia de un sistema de gestión de ideas de los clientes, es posible rechazar la hipótesis nula de igualdad de medias y asumir que existen diferencias significativas.

Adicionalmente hay que señalar que la prueba de Kruskal-Wallis señala que en el caso del nivel de innovaciones de organización en los últimos tres años (INNOV7) no se han encontrado evidencias suficientes que relacionen esta variable con la orientación al mercado, por lo que no es posible confirmar el resultado obtenido con la prueba de Brown-Forsythe.

Tabla 6. Pruebas robustas de igualdad de las medias y pruebas no paramétricas

\begin{tabular}{|c|c|c|c|c|c|c|}
\hline & \multicolumn{4}{|c|}{ Brown- Forsythe } & \multicolumn{2}{|c|}{ Kruskal-Wallis } \\
\hline & Estadístico $^{\mathrm{a}}$ & gl1 & gl2 & Sig. & Chi cuadrado & Sig \\
\hline INNOV & & $\cdot$ & & & 9,801 & $0,020 * *$ \\
\hline ACT_INT & 2,444 & 3 & 22,395 &, $091 *$ & 7,113 & $0,068^{*}$ \\
\hline INNOV1 & & . & & & 5,588 & 0,133 \\
\hline INNOV2 & & . & & & 12,909 & $0,005 * *$ \\
\hline INNOV7 & 2,389 & 3 & 32,365 &, $087 *$ & 5,596 & 0,133 \\
\hline CON_CLI & 4,739 & 3 & 20,420 &, $012 * *$ & 12,913 & $0,005 * *$ \\
\hline
\end{tabular}

a. Distribuidos en F asintóticamente.

Fuente: Elaboración propia

Los resultados obtenidos demuestran que existen diferencias estadísticamente significativas entre las cooperativas con diferente grado de orientación al mercado en relación al perfil del gerente (ACT_INT) y el conocimiento de sus clientes (CON_CLI).

Una vez detectadas las variables en las que existen diferencias significativas, se realizan las pruebas post-hoc DMS (también conocida como LSD, Least Significant Difference) y T2 de Tamhane para determinar la naturaleza y los grupos implicados en ellas. En la Tabla 7 se muestra un resumen de los resultados obtenidos. 
Tabla 7. Resumen resultados de pruebas post-hoc

\begin{tabular}{|c|c|c|c|c|c|}
\hline & & Descripción & $\begin{array}{l}\text { Niveles con diferencias } \\
\text { significativas }\end{array}$ & $\begin{array}{l}\text { Nivel con } \\
\text { mayor } \\
\text { valor }\end{array}$ & $\begin{array}{c}\text { Nivel de } \\
\text { significación }\end{array}$ \\
\hline \multirow[t]{4}{*}{$\begin{array}{l}\text { Perfil del } \\
\text { empresario }\end{array}$} & \multirow{2}{*}{ RIGIDEZ } & \multirow[t]{2}{*}{$\begin{array}{l}\text { Rigidez empresarial, resistencia al } \\
\text { cambio }\end{array}$} & 61-76 y Más de 85 & $\begin{array}{l}\text { Más de } \\
85\end{array}$ & $0.041 * *$ \\
\hline & & & 76-85 y Más de 85 & $\begin{array}{l}\text { Más de } \\
85\end{array}$ & $0.02 * *$ \\
\hline & \multirow{2}{*}{ AUSE_AG } & \multirow[t]{2}{*}{$\begin{array}{l}\text { Ausencia de agresividad } \\
\text { empresarial }\end{array}$} & $\begin{array}{c}\text { Menos de } 61 \text { y Más de } \\
85\end{array}$ & $\begin{array}{l}\text { Más de } \\
85\end{array}$ & $0.064 *$ \\
\hline & & & 61-76 y Más de 85 & $\begin{array}{l}\text { Más de } \\
85\end{array}$ & $0.008 * *$ \\
\hline \multirow[t]{12}{*}{ Innovación } & \multirow{4}{*}{ INNOV3 } & \multirow[t]{4}{*}{ Innovaciones de producto } & Menos de 61 y $76-85$ & $76-85$ & $0.087 *$ \\
\hline & & & $\begin{array}{l}\text { Menos de } 61 \text { y Más de } \\
85\end{array}$ & $\begin{array}{l}\text { Más de } \\
85\end{array}$ & $0.001 * *$ \\
\hline & & & 61-76 y Más de 85 & $\begin{array}{l}\text { Más de } \\
85\end{array}$ & $0.022 * *$ \\
\hline & & & 76-85 y Más de 85 & $\begin{array}{l}\text { Más de } \\
85\end{array}$ & $0.03 * *$ \\
\hline & \multirow{3}{*}{ INNOV4 } & \multirow[t]{3}{*}{$\begin{array}{l}\text { Esfuerzos realizados en } \\
\text { innovaciones de producto }\end{array}$} & $\begin{array}{l}\text { Menos de } 61 \text { y Más de } \\
85\end{array}$ & $\begin{array}{l}\text { Más de } \\
85\end{array}$ & $0.019 * *$ \\
\hline & & & 61-76 y Más de 85 & $\begin{array}{l}\text { Más de } \\
85\end{array}$ & $0.059 *$ \\
\hline & & & 76-85 y Más de 85 & $\begin{array}{l}\text { Más de } \\
85\end{array}$ & $0.043 * *$ \\
\hline & \multirow{2}{*}{ INNOV5 } & \multirow[t]{2}{*}{ Innovaciones de proceso } & $\begin{array}{c}\text { Menos de } 61 \text { y Más de } \\
85\end{array}$ & $\begin{array}{l}\text { Más de } \\
85\end{array}$ & $0.014 * *$ \\
\hline & & & 76-85 y Más de 85 & $\begin{array}{l}\text { Más de } \\
85\end{array}$ & $0.057 *$ \\
\hline & INNOV7 & Innovaciones de organización & 61-76 y Más de 85 & $\begin{array}{l}\text { Más de } \\
85\end{array}$ & $0.075^{*}$ \\
\hline & \multirow{2}{*}{ INNOV8 } & \multirow[t]{2}{*}{$\begin{array}{l}\text { Esfuerzos en innovaciones de } \\
\text { organización }\end{array}$} & $\begin{array}{c}\text { Menos de } 61 \text { y Más de } \\
85\end{array}$ & $\begin{array}{l}\text { Más de } \\
85\end{array}$ & $0.049 * *$ \\
\hline & & & 61-76 y Más de 85 & $\begin{array}{l}\text { Más de } \\
85\end{array}$ & $0.032 * *$ \\
\hline \multirow[t]{3}{*}{$\begin{array}{l}\text { Conocimiento } \\
\text { del entorno }\end{array}$} & CON_CLI & $\begin{array}{l}\text { Grado de conocimiento de la } \\
\text { cooperativas sobre clientes } \\
\text { exteriores }\end{array}$ & $\begin{array}{l}\text { Menos de } 61 \text { y Más de } \\
85\end{array}$ & $\begin{array}{l}\text { Más de } \\
85\end{array}$ & $0.087 *$ \\
\hline & \multirow[b]{2}{*}{ CON_COMP } & \multirow{2}{*}{$\begin{array}{l}\text { Grado de conocimiento de la } \\
\text { cooperativa sobre competidores }\end{array}$} & Menos de 61 y $76-85$ & $76-85$ & $0.70 *$ \\
\hline & & & $\begin{array}{c}\text { Menos de } 61 \text { y Más de } \\
85\end{array}$ & $\begin{array}{l}\text { Más de } \\
85\end{array}$ & $0.12 * *$ \\
\hline
\end{tabular}

Fuente: Elaboración propia

\subsection{Análisis de conglomerados}

El análisis de conglomerados se realiza para detectar relaciones subyacentes en los datos a partir de la agrupación de los individuos de la muestra en grupos homogéneos. El número de grupos ha sido obtenido a partir de un análisis utilizando métodos jerárquicos y se ha establecido en tres. Tomando como base esta información y utilizando método no jerárquicos se realiza la agrupación final de los individuos de la muestra, determinando los centroides finales de los conglomerados (Tabla 8). 
Tabla 8. Centros de los conglomerados finales

\begin{tabular}{|l|r|r|r|}
\hline \multirow{2}{*}{} & \multicolumn{3}{|c|}{ Conglomerado } \\
\cline { 2 - 4 } & 1 & 2 & \multicolumn{1}{|c|}{3} \\
\hline Dimensión & 1,80 & 2,29 & 2,45 \\
FORMACION & 1,70 & 3,96 & 4,09 \\
INNOV &, 40 &, 75 &, 27 \\
PESO_EXP & 1,00 &, 75 &, 82 \\
OM & 3,10 & 3,67 & 1,55 \\
\hline Casos & 10 & 24 & 11 \\
\hline \multicolumn{3}{|c|}{ Fuente: Elaboración propia }
\end{tabular}

El grupo 1 -formado por 10 cooperativas- es el que presenta una menor dimensión empresarial con directivos de menor formación académica que no consideran innovadora a la cooperativa. No obstante estas cooperativas se sitúan en una posición intermedia-alta respecto a la orientación al mercado y exportan más del $50 \%$ de su producción.

El grupo 2 está formado por 24 cooperativas de tamaño pequeño y mediano, sus directivos cuentan con educación superior y consideran en su mayoría a la cooperativa como innovadora. El porcentaje de ventas en el extranjero es también muy elevado y presentan la mayor orientación al mercado de todos los segmentos.

El grupo 3, formado por 11 entidades, está integrado por las cooperativas de mayor tamaño que a su vez cuentan con los directivos mejor formados de la muestra, con educación universitaria y cursos de postgrado o especialización. Sin embargo, son las cooperativas que no se consideran innovadoras y que presentan un peor resultado en relación a la orientación al mercado.

Para establecer si existen diferencias significativas entre los factores de competitividad de las cooperativas que forman parte de los distintos grupos formados, se realiza un análisis de varianza (Tabla 9). El ANOVA señala que existen diferencias significativas en base a la dimensión (con un nivel de confianza del 10\%) y respecto a la formación de los directivos, la innovación y la orientación al mercado (con un nivel de confianza del 5\%). 
Tabla 9. ANOVA

\begin{tabular}{|l|r|r|r|r|r|r|}
\hline & \multicolumn{2}{|c|}{ Conglomerado } & \multicolumn{2}{c|}{ Error } & \multirow{2}{*}{ F } & \multirow{2}{*}{ Sig. } \\
\cline { 2 - 6 } & Media cuadrática & $\mathrm{gl}$ & Media cuadrática & $\mathrm{gl}$ & & \\
\hline Dimensión & 1,246 & 2 &, 507 & 42 & 2,459 &, 098 \\
FORMACION & 20,639 & 2 &, 475 & 42 & 43,412 &, 000 \\
INNOV & 1,015 & 2 &, 216 & 42 & 4,692 &, 014 \\
PESO_EXP &, 221 & 2 &, 146 & 42 & 1,511 &, 233 \\
OM & 17,009 & 2 &, 309 & 42 & 55,118 &, 000 \\
\hline
\end{tabular}

Las pruebas F sólo se deben utilizar con una finalidad descriptiva puesto que los conglomerados han sido elegidos para maximizar las diferencias entre los casos en diferentes conglomerados. Los niveles críticos no son corregidos, por lo que no pueden interpretarse como pruebas de la hipótesis de que los centros de los conglomerados son iguales.

Fuente: Elaboración propia

\section{CONCLUSIONES}

El estudio de los factores de competitividad es una necesidad actual de todos los agentes que operan en el mercado. Las cooperativas agroalimentarias por sus características particulares de estructura de la propiedad y gobierno necesitan un análisis específico para determinar el alcance y la repercusión que dichos factores tienen en su actividad.

Este trabajo contribuye a ampliar el conocimiento existente sobre este área de la gestión, aportando evidencia empírica sobre el caso particular de una muestra representativa de cooperativas citrícolas españolas. En base a la muestra analizada ha sido posible proponer una clasificación de las cooperativas en función del grado de orientación al mercado mostrado en base a la escala MARKOR.

En relación al primer objetivo del trabajo, los resultados ponen de manifiesto la existencia de relaciones significativas entre la orientación al mercado en las cooperativas y la innovación que éstas llevan a cabo, sea ésta de tipo producto, proceso u organizativa. En referencia a las innovaciones de producto, se detectan diferencias significativas entre todas las tipologías de cooperativas realizadas en función del grado de orientación al mercado en relación a las de mayor grado de orientación así como entre aquellas que obtienen puntuaciones inferiores a 61 y las que se sitúan entre 76 y 85 . En todos los casos, un mayor grado de orientación al mercado está asociado a mayores niveles de innovación en producto.

La observación anterior queda corroborada al observar los esfuerzos realizados en materia de innovación de producto, al obtener respuestas semejantes: las cooperativas 
citrícolas con mayor orientación al mercado se diferencian significativamente del resto de categorías, señalando un esfuerzo más importante en este aspecto o una mayor concienciación sobre este aspecto de la gestión.

Las innovaciones en proceso y en organización registran también comportamientos diferenciados en función del grado de orientación al mercado. Se constata el patrón anterior, a mayor orientación al mercado, mayor grado de innovaciones en proceso o en organización, pero en estos dos casos el número de diferencias significativas es menor. Únicamente es posible señalar diferencias significativas entre cooperativas que se sitúan en posiciones extremas respecto a la orientación al mercado, razón por la que se podría señalar que en este ámbito, innovaciones de proceso y organización, existe aún un grado amplio de desarrollo en el sector cooperativo.

El perfil del gerente/director general/director comercial también se ha revelado como un factor determinante en el grado de orientación al mercado que muestra una cooperativa. Las cooperativas con mayor grado de orientación al mercado muestran diferencias significativas respecto al resto de tipologías definidas en la postura adoptada por su directivo ante el riesgo y la agresividad del entorno. Los directivos muestran mayor cautela en la toma de decisiones en aquellas cooperativas más orientadas al mercado. Estas conclusiones se alinean con las obtenidas en estudios previos (Kajalo y Lindblom, 2015; Cai et al, 2015, entre otros) referidos a la relación entre la orientación al mercado y la orientación emprendedora (que se compone de tres dimensiones: innovación, proactividad y asunción de riesgos).

La hipótesis relativa a la relación de la dimensión con el grado de orientación al mercado no ha podido ser contrastada por la falta de variabilidad suficiente en la muestra para poder llevar a cabo el análisis.

El segundo objetivo del trabajo hace referencia al establecimiento de una clasificación de las cooperativas de la muestra atendiendo a su desempeño en los siguientes factores de competitividad: dimensión, formación, orientación al mercado, innovación e internacionalización. El análisis de conglomerados (clúster) ha determinado que existen tres grupos homogéneos a nivel interno y que éstos presentan a su vez suficientes diferencias entre ellos para ser distinguidos.

Un primer grupo se refiere a las cooperativas más pequeñas, con un menor nivel de formación en su staff directivo y que, a pesar de no considerarse innovadoras, se sitúan en una 
posición intermedia-alta en cuanto a la orientación al mercado y consiguen buenos resultados en mercados internacionales, exportando más del $50 \%$ de su producción.

El segundo grupo es el más numeroso, con cooperativas de tamaño pequeño y mediano, la dirección cuenta con educación superior y se consideran innovadoras. Son las cooperativas que mayor grado de orientación al mercado presentan en la muestra.

El tercer grupo es singular, está integrado por las cooperativas de mayor dimensión y cuenta con la dirección mejor formada (educación superior y cursos de postgrado y/o especialización). No obstante, no se consideran innovadoras en su mayoría y son las que peor resultado obtienen en relación a la orientación al mercado. Respecto a este grupo parece oportuno indicar que debido a que tanto la innovación como la orientación son evaluadas por el propio directivo, una posible razón que explique este resultado sea un nivel excesivo de autocrítica en el momento de evaluar a la cooperativa o un mayor nivel de exigencia.

Esta consideración enlaza con una de las limitaciones que presenta el trabajo y es la derivada del sesgo que introduce en los datos la autoevaluación de la cuestión de la innovación y la orientación al mercado por parte de los directivos, lo cual puede afectar a la calidad de los resultados y conclusiones obtenidas. En segundo lugar el limitado número de entidades que cumplen las condiciones especificadas condiciona los análisis, dificultando o incluso impidiendo obtener conclusiones sobre algunas de las hipótesis planteadas. Una ampliación de la muestra a otros subsectores agrarios podría solventar esta cuestión si bien ampliaría la heterogeneidad de la muestra, introduciendo otra problemática.

\section{BIBLIOGRAFÍA}

ÁLVAREZ, L.I.; SANTOS M.L. y VÁZQUEZ, R. (2001) El concepto de orientación al mercado: perspectivas, modelos y dimensiones de análisis. Universidad de Oviedo.

AMADIEU, P. y VIVIANI, J.L. ( 2010) Intangible Effort and Performance: The Case of the French Wine Industry. Agribusiness, Vol. 26, No 2, pp. 280-306.

ARAGÓN, A. y RUBIO, A. (2005) Factores asociados con el éxito competitivo de las PYMEs industriales en España. Universia Business Review, No 8, pp. 38-51.

ARCAS, N. (2002) Contribución de las cooperativas agrarias de segundo grado a la orientación al mercado de sus cooperativas asociadas: efectos en el desempeño de la relación. CIRIEC-España, Revista de Economía Pública, Social y Cooperativa, No 41, pp. 139-161. 
BIJMAN, J. (2010) Agricultural cooperatives and market orientation: a challenging combination? En Market orientation: transforming food and agribusiness around the customer. Lindgreen, A., Hingley, M., Custance, P., Aldershot: Gower Publishing, p. 119136.

BILKEY, W. J. y TESAR, G. (1977) The export behavior of smaller sized Wisconsin manufacturing firms. Journal of International Business Studies, № 3, pp. 93-98.

BOSO, N.; CADOGAN, J.W. y STORY, V.M. (2013) Entrepreneurial orientation and market orientation as drivers of product innovation success: a study of exporters from a developing country. International Small Business Journal, Vol. 31, № 1, pp. 57-81.

CAI, L; LIU, Q.; ZHU, X.M. y DENG, S.L. (2015) Market orientation and technological innovation: the moderating role of entrepreneurial support policies. International Entrepreneurship and Management Journal, Vol. 11, № 3, pp. 645-671.

CAMBRA, J. y FUSTER, A. (2004) Factores determinantes de la orientación a largo plazo de las relaciones socio-cooperativa: un estudio empírico en el sector agroalimentario aragonés. Comunicación presentada en el XVIII Congreso Anual y XIV Congreso Hispano-Francés de AEDEM. Ourense, 2-4 de Junio.

CAMBRA, J. y FUSTER, A. (2005) Importancia de la medición del grado de orientación al mercado en el sector cooperativista como respuesta al nuevo marco competitivo. Investigaciones Europeas de Dirección y Economía de la Empresa, Vol. 11, № 3, pp. 6783.

CAMBRA, J.J.; FRAJ, E., MELERO, I.; SESÉ, F.J. y VÁZQUEZ, R. (2011) Orientación al mercado y al aprendizaje en el sector vinícola aragonés. Documento de trabajo Fundación Economía Aragonesa (FUNDEAR), Nº 58, pp. 1-76.

CASTAÑO-MARTÍNEZ, M.S. (2012) Product innovation and R\&D policy: the case of the transformation industries in developed and developing. International Entrepreneurship and Management Journal, Vol. 8, № 4, pp. 421-436.

CAVUSGIL, S.T. y GODIWALLA, Y.M. (1982) Decision-making for International Marketing: A Comparative Review, Management Decision, Vol. 20, № 4, pp. 47-54.

CAVUSGIL, S. T. (1984) Organizational characteristics associated with export activity. Journal of Management Studies, Vol. 21, № 1, pp. 3-22.

DAI, L.; MAKSIMOV, V.: GILBERT, B.A. y FERNHABER, S.A. (2014) Entrepreneurial orientation and international scope: The differential roles of innovativeness, proactiveness and risk-taking. Journal of Business Venturing, Vol. 29, № 4, pp. 511-524. 
DEL RÍO, M.L. y VARELA, M.C. (2006) Características de los directivos y rendimiento exportador en las pymes españolas. ESIC Market, N 125 , pp. 223-254.

DESHPANDÉ, R.; FARLEY, J.U. y WEBSTER, F.E. (1993) Corporate culture, customer orientation and innovativeness in Japanese Firms: A Quadrad Analysis. Journal of Marketing, № 57 , pp. 23-57

FOOD AND AGRICULTURE ORGANIZATION OF THE UNITED NATIONS (2013) FAOSTAT database. Disponible en: http://faostat3.fao.org/home/E.

FIAB, Federación Española de Industrias de la Alimentación y Bebidas (2013).

GARCÍA, E. y ARANDA, M. (2001) Problemas existentes en la profesionalización de la gestión en la sociedad cooperativa. Investigaciones Europeas de Dirección y Economía de la Empresa, Vol. 7, № 3, pp. 139-154.

GÓMEZ, J.E.; RIALP, J. y LLONCH, J. (2009) Influencia de la Orientación al Mercado en la Función Empresarial; Su Impacto en la Capacidad de Innovación y en los Resultados de la PYME Española. Revista Internacional de la Pequeña y Mediana Empresa, № 1, pp. 4647.

GONZÁLEZ-BENITO, O.; MUÑOZ-GALLEGO, P.A. y GARCÍA-ZAMORA, E. (2015) Entrepreneurship and market orientation as determinants of innovation: the role of business size. International Journal of Innovation Management, $\mathrm{N}^{\circ} 19$.

GRINSTEIN, A. (2008) The Effect of Market Orientation and its Components on Innovation Consequences: a Meta Analysis. Journal of the Academy of Marketing Science, № 36, pp. 166-173.

GRUNERT, K. G.; JEPPESEN, L.F.; JESPERSEN, K.R.; SONNE, A.M., HANSEN, K.; TRONDSEN, T. y YOUNG, J.A. (2005) Market orientation of value chains: A conceptual framework based on four case studies from the food industry. European Journal of Marketing, Vol. 39, pp. 428-455.

GUPTA, A.K. y GOVINDARAJAN, V. (1984) Business unit strategy, managerial characteristics and business unit effectiveness at strategy implementation. Academy of Management Journal, Vol. 27, No 1, pp. 25-41.

GUZMÁN, J.; LAFUENTE, C. y POZA, C. (2014) Characterization of business in terms of functional dependence and productive dependence. An application to six Spanish regions. Revista de Estudios Regionales, Vol. 101, No 1, pp. 135-1625.

HAIR, J.F.; ANDERSON, R.E.; TATHAM, R.L. y BLACK, W.C. (1999) Análisis multivariante. $5^{\text {a }}$ ed. Prentice-Hall. 
HAN, J.; KIM, N. y SRIVASTAVA, R. (1998) Market orientation and organizational performance: is innovation the missing link?, Journal of Marketing, Vol. 62, October, pp. $30-45$.

HE, X. y WEI, Y. (2011) Linking market orientation to international market selection and international performance. International Business Review, Vol. 20, № 5, pp. 535-546.

HERNANDEZ-ESPALLARDO, M. y ARCAS-LARIO, N. (2003) The Effects of Authoritative Mechanisms of Coordination on Market Orientation in Asymmetrical Channel Partnerships. International Journal of Research in Marketing, Vol. 20, $\mathrm{N}^{\mathrm{o}}$ 2, pp. 133-152.

JAWORSKI, B. J. y KOHLI, A. K. (1996) Market Orientation: Review, Refinement and Roadmap. Journal of Market Focused Management, No 1, pp. 119-135.

JULIÁ, J.F.; LAJARA-CAMILLERI, N. y MELIA, E. (2009) Factores de competitividad en las cooperativas agrarias valencianas. La orientación al mercado y las alianzas empresariales en La gestión de empresas de economía social. Ed. Thomson.

JULIÁ, J.F.; GARCÍA, G.; MELIÁ, E. y GALLEGO, L.P. (2010) Los factores de competitividad de las cooperativas líderes en el sector agroalimentario europeo. Ed. Fundación CAJAMAR, Almería.

JULIÁ, J.F. y MARÍ, S. (2002) Agricultura y desarrollo rural. Contribuciones de las cooperativas agrarias. CIRIEC-España, Revista de Economía Pública, Social y Cooperativa, $\mathrm{N}^{\mathrm{o}} 41$, pp. 25-52.

KAJALO, S. y LINDBLOM, A. (2015) Market orientation, entrepreneurial orientation and business performance among small retailers. International Journal of Retail and Distribution Management, Vol. 43, № 7, pp. 580-596.

KOHLI, A. K. y JAWORSKI, B.J. (1990) Market Orientation: The Construct, Research Propositions, and Managerial Implications. Journal of Marketing, № 54, pp. 1-18.

KYRIAKOPOULOS, K. y VAN BEKKUM, O.F. (1999) Market orientation of European Agricultural Cooperatives: Strategic and Structural Issues, IX European Congress of Agricultural Economists, Varsovia, Polonia, 24-28 Agosto.

KYRIAKOPOULOS, K.; MEULENBERG, M. y NILSSON, J. (2004) The impact of cooperative structure and firm culture on market orientation and performance. Agribusiness, Vol. 20, No 4, pp. 379-396.

LADO, N. y MAYDEU-OLIVARES, A. (2001) Exploring the link between market orientation and innovation in the European and US insurance markets. International Marketing Review, Vol. 18, № 2, pp. 130-144. 
LIU, H. (1995) Market orientation and firm size: en empirical examination in UK firms. European Journal of Marketing, Vol. 29, № 1, pp. 57-72.

LONGINOS, J.; ARCAS, N.; MARTÍNEZ, I.M. y OLMEDO, I. (2012) Transparencia, Gobierno Corporativo y Participación: claves para la implantación de un código de conducta en empresas de economía social. REVESCO. Revista de Estudios Cooperativos, No 108, pp. 86-112. DOI: http://dx.doi.org/10.5209/rev_REVE.2012.v18.39588.

MAGRAMA, Ministerio de Agricultura, Alimentación y Medio Ambiente (2012) Anuario de Estadística.

MARÍ-VIDAL, S.; LAJARA-CAMILLERI, N. y SERVER, R.J. (2013) La formación en las sociedades cooperativas agrarias como factor clave de competitividad en un contexto de concentración e internacionalización de los mercados. Interciencia, Vol. 38, № 2, pp. 112 120.

MARTÍN ARMARIO, J. (2003) Orientación al mercado y proceso de internacionalización de las empresas. Tesis doctoral.

http://www.biblioteca.uma.es/bbldoc/tesisuma/1669952x.pdf.

MARTÍN-ARMARIO, J.; MARTÍN-RUIZ, D. y MARTÍN-ARMARIO, E. (2008) Market Orientation and internationalization in small and medium-sized enterprises, Journal of Small Business Management, Vol. 46, № 4, pp. 85-511.

MELIA-MARTI, E. y MARTINEZ-GARCIA, A. M. (2015) Characterization and analysis of cooperative mergers and their results. Annals of Public and Cooperative Economics. Vol $86, \mathrm{~N}^{\mathrm{o}} 3$, pp. 479-504.

MONTEGUT, Y.; CRISTOBAL, E. y MARIMON, F. (2007) Orientación al mercado en las almazaras cooperativas: el caso de Cataluña. La sociedad cooperativa, № 34, pp. 29-34.

MOZAS, A. y RODRÍGUEZ, J. (2003) La formación de los recursos humanos de las cooperativas agrarias y la nueva economía: un estudio empírico. Boletín Económico del ICE, Información Comercial Española, № 2776, pp. 9-19.

NARVER, J. y SLATER, S. (1990) The Effect of Market Orientation on Business Profitability. Journal of Marketing, $\mathrm{N}^{\circ}$ 54, pp. 20-35.

OSCAE, Observatorio Socioeconómico del Cooperativismo Agroalimentario Español (2013) http://www.agro-alimentarias.coop/cooperativismo_en_cifras.

PEÑA, D. (2010) Regresión y diseño de experimentos. Alianza Editorial, Madrid.

RIVERA, J. (1999) La orientación al mercado como estrategia competitiva de la organización. Investigaciones Europeas de Dirección y Economía de la Empresa, Vol. 5, $\mathrm{N}^{\mathrm{o}} 3$, pp. $107-126$ 
SENISE, O. (2003) La orientación al mercado en el sector oleícola andaluz: un análisis a partir de las cooperativas de segundo y ulterior grado. Ed. Analistas Económicos de Andalucía.

SUH, Y. y KIM, M. (2014) Internationally leading SMEs vs. internationalized SMEs: Evidence of success factors from South Korea. International Business Review, $\mathrm{N}^{\mathrm{o}} 23$, pp. 115-129.

TUOMINEN, M.; MÖLLER K. y RAJALA A. (1997) Marketing Capability: A Nexus of Learning-Based Resources and a Prerequisite for Market Orientation in 26th European Marketing Academy (EMAC) Annual Conference Proceedings, Warwick, UK, May. Vol.1, pp. 1220-1240.

VÁZQUEZ, R.; POLO, Y. y BELLO, L. (1999) Recientes aportaciones del marketing a la cultura corporativa y a la obtención de ventajas competitivas. Papeles de Economía Española, № 78 , pp. 190-211.

\section{ANEXO}

Escala Orientación al mercado utilizada (Arcas, 2002)

\begin{tabular}{l|l|l|l|l|l|} 
& $\begin{array}{c}\text { Muy en } \\
\text { desacurdo }\end{array}$ & $\begin{array}{c}\text { Algo en } \\
\text { desacuerdo }\end{array}$ & Indiferente & $\begin{array}{c}\text { Algo de } \\
\text { acuerdo }\end{array}$ & $\begin{array}{l}\text { Muy de } \\
\text { acuerdo }\end{array}$ \\
\hline $\begin{array}{l}\text { Tratamos de conocer con exactitud los productos que los } \\
\text { clientes demandarán en el futuro }\end{array}$ & & & & & \\
\hline $\begin{array}{l}\text { Se trata de acceder periódicamente a información sobre el } \\
\text { mercado }\end{array}$ & & & & & \\
\hline $\begin{array}{l}\text { Somos capaces de detectar rápidamente cambios en las } \\
\text { preferencias de los clientes }\end{array}$ & & & & & \\
\hline $\begin{array}{l}\text { Analizamos, al menos anualmente, la opinión de los clientes } \\
\text { sobre la calidad de los productos }\end{array}$ & & & & & \\
\hline $\begin{array}{l}\text { Detectamos con rapidez los cambios que se producen en el } \\
\text { entorno (legislación, competencia, tecnología...) }\end{array}$ & & & & & \\
\hline $\begin{array}{l}\text { Analizamos cómo los cambios en el entorno (legislación, } \\
\text { competencia, tecnología...) afectan a los clientes }\end{array}$ & & & & & \\
\hline $\begin{array}{l}\text { Respondemos de forma rápida a las acciones de la } \\
\text { competencia }\end{array}$ & & & & & \\
\hline $\begin{array}{l}\text { Revisamos periódicamente los productos para asegurarnos de } \\
\text { que están en línea con las necesidades de los clientes }\end{array}$ & & & & & \\
\hline $\begin{array}{l}\text { Planificamos entre varios departamentos la respuesta a los } \\
\text { cambios en el entorno (legislación, competencia, }\end{array}$ & & & & & \\
tecnología...)
\end{tabular}




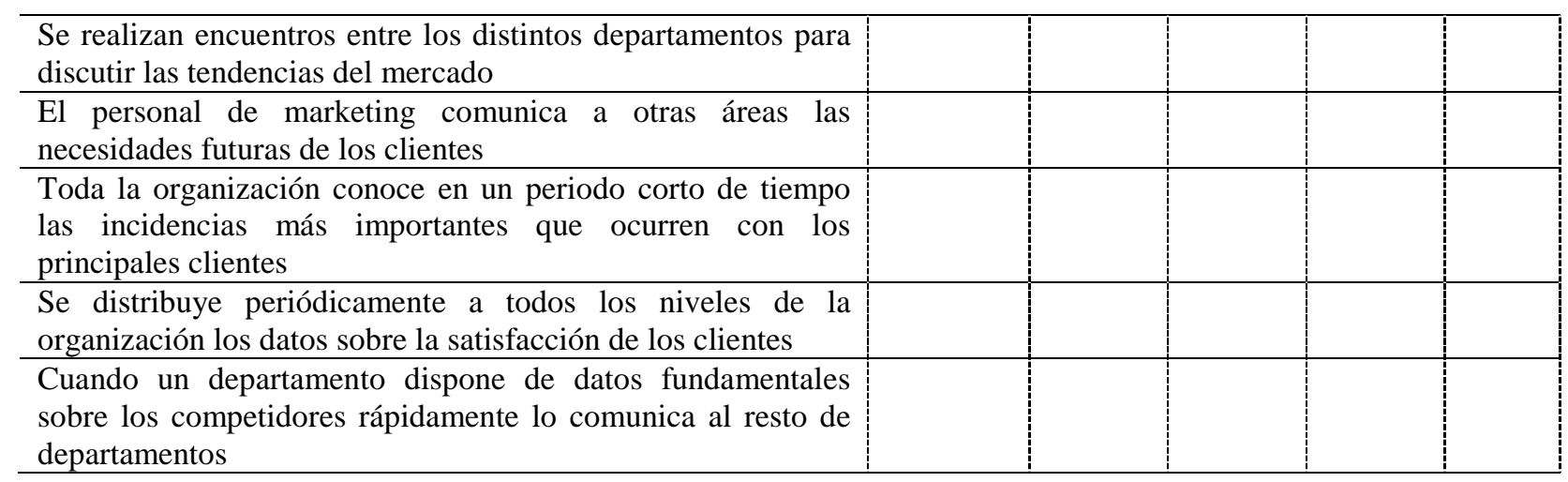

